\title{
Über die Evolution der polnischen Lehrpläne und Richtlinien im Fremdsprachenunterricht
}

\author{
On the evolution of Polish syllabi \\ and core curricula in foreign language teaching
}

\begin{abstract}
The first part of the article is devoted to the terminology connected with the notions of core curriculum and syllabi. With reference to the subject, a five-level model of working on documents is presented. After, there follows a historical outline on the evolution of documents, from the national school curriculum to the first core curricula of general education, with reference to foreign language teaching at various stages of education, which was prepared based of the analysis of legal documents. The next chapter shows a syllabus from the present-day perspective of education law, and presents its functions. It describes the results of pilot questionnaires on the functions and role of German teaching syllabi in the Polish educational system in the context of previous theoretical considerations. The article concludes with ideas on leading and optimizing the role of contemporary curricula documents.
\end{abstract}

KEYWORDS: national curriculum, core curricula, syllabi, optimizing education.

SCHLÜSSELWORTE: zentraler Lehrplan, Richtlinien, Curriculum, Optimalisierung der Bildungsprozesse.

\section{EINLEITUNG}

Was bezweckt die Fremdsprachenvermittlung? Welche Aufgaben stellt man dem modernen Fremdsprachenunterricht? Solche Fragen sind nicht nur theoretischer Natur, weil sie sowohl den Politikern als auch Praktikern als 
Leitgedanke ihres Handelns dienen. Wie Westphalen (1985: 9) feststellt: „Im Zentrum aller Überlegungen steht dabei stets der Fragenkomplex: Welche Inhalte, Fähigkeiten und Fertigkeiten sollen den nachfolgenden Generationen zu welchem Zwecke vermittelt werden?" In Bezug darauf kann die Problematik der curricularen Planung im Bereich der Fremdsprachendidaktik in Polen im historischen Abriss sowie auch aus der heutigen Perspektive angegriffen werden. Das Ziel des Beitrags ist zu schildern, wie sich die wichtigsten Steuerungsinstrumente der didaktischen Prozesse der Fremdsprachenvermittlung, nämlich Lehrpläne und Rahmenrichtlinien änderten.

Der erste Teil widmet sich der terminologischen Klärung der Begriffe: Richtlinien, Lehrpläne, Curricula. Anschließend wird zum 5-Phasen-Modell der Lehrplanarbeit übergegangen. Weiterhin wird ein historischer Überblick über curriculare Dokumente von zentralen und produktorientierten Lehrplänen bis zu der Entstehung der ersten Richtlinien für die Allgemeinbildung gegeben, fokussiert auf die neueren Sprachen in verschiedenen Bildungsstufen. Auf alle Verordnungen für die Richtlinien, die analysiert wurden, wird im Text kurz mit der Angabe des Jahres hingewiesen, genauere Informationen sind im Literaturverzeichnis zu finden. Im Fokus des weiteren Abschnitts steht der Begriff des Lehrplans in dem heutigen gesetzlichen Zustand und hinsichtlich seiner Funktion. Im Kontext der früheren theoretischen Überlegungen werden als nächstes die Ergebnisse einer Pilotuntersuchung zum Thema „Funktion und Bedeutung der Lehrpläne im DaFUnterricht im polnischen Schulsystem" präsentiert. Im letzten Teil werden Schlussfolgerungen zur Funktion der heutigen curricularen Dokumente formuliert.

\section{RICHTLINIEN, LEHRPLÄNE, CURRICULA - TERMINOLOGISCHE KLÄRUNG}

Die Fremdsprachenvermittlung verläuft im Allgemeinen in drei grundsätzlichen Phasen, die als Planung, Durchführung und Bewertung von Unterricht zu bezeichnen sind (Edmondson \& House 2006: 292). „Planung als anthropologische Grundkategorie im Sinne von Karl Jaspers bedeutet für den pädagogischen Bereich die Antizipation und Strukturierung von Prozessen und Produkten zukünftigen Handelns" (Funk 2016: 151), sie gehört also zu den Grundkomponenten der pädagogischen Tätigkeit. Im Fokus der Betrachtungen dieses Artikels stehen Richtlinien für den Fremdsprachenunterricht in Polen und Lehrpläne für den DaF-Unterricht, die ein vorrangiger Bestandteil der prädidaktischen Etappe bilden. In der methodischen Phase dieser Etappe werden nämlich diese obligatorischen und unentbehrlichen 
Dokumente entwickelt, an die die Grunderwartungen, den Unterrichtsprozess zu steuern, gestellt werden (Pfeiffer 2001: 34). An dieser Stelle muss man sich der terminologischen Klärung widmen und die beiden Begriffe voneinander abgrenzen, weil in der wissenschaftlichen und internationalen Diskussion viele Bezeichnungen auftreten, die synonymisch verwendet werden, von denen z. B. Richtlinien, Rahmenrichtlinien, Curricula, Lehrpläne oder Bildungspläne zu nennen sind. Die Autoren solcher Dokumente halten sich nicht immer an die terminologische Unterscheidung, deswegen ist es in einigen Fällen unmöglich eine Abgrenzung zu setzen. Aus semantischen und begrifflichen Gründen wäre es wichtig, die im Titel des Artikels erwähnten Grundbegriffe und ihre Bedeutung in der polnischen Fremdsprachendidaktik näher zu diskutieren (Neuner 2001: 799). Was aber einen wesentlichen Unterschied zwischen den oben erwähnten Bezeichnungen macht,

sind nicht ihre Inhalte, nicht die didaktischen Kategorien, nach denen sie geordnet sind (Lernziele, Lerninhalte, Lernorganisation, Erfolgskontrolle), auch nicht die didaktischen Prinzipien, nach denen die Strukturierung des Unterrichts erfolgen sollte (z.B. Anschaulichkeit, Exemplarität, Wissenschaftsorientierung), sondern vor allem

- die Funktionen, die sie als Baupläne oder Bausteine des Unterrichts erfüllen sollen,

- die Entscheidungskriterien und -prozesse, nach denen sie entstanden und aus denen sie hervorgegangen sind,

- der Grad der Verbindlichkeit, den sie beanspruchen. (Westphalen 1985: 12)

Die polnischen Richtlinien sind als obligatorische Zusammenstellung von Bildungszielen und -inhalten, sowie auch Fertigkeiten, die als allgemeine und ausführliche Anforderungen im Bereich des Wissens und Könnens, die die Schüler nach dem Abschluss eines bestimmten Schultyps erworben haben, zu verstehen. Diese Ziele und erwartete Leistungen müssen in den internen Lehrplänen der Schule berücksichtigt werden und es ermöglichen, Bewertungskriterien sowie auch Prüfungsanforderungen festzulegen (Prawo oświatowe, art. 4). „Lehrziele, Lehrstoffe und Inhalte werden dabei nicht auf die einzelnen Jahrgänge verteilt (Progression), sondern in der Form von Anforderungen zum Erreichen des jeweiligen Niveaus angegeben" (Neuner 2001: 799). Wichtig ist zu erwähnen, dass Richtlinien Freiräume in der Gestaltung unterrichtlicher Lehr-Lernprozesse gewährleisten sollten (Christ 2016: 58). Lehrpläne dagegen bestimmen „die fachspezifischen Aufgaben, Ziele, Inhalte, die konkrete Lerndauer und Progression, die Unterrichtsgestaltung und -organisation sowie die Verfahren der ebenfalls fachspezifisch angelegten Lernerfolgsüberprüfung" (Bausch 2003: 112). 
Auf der Ebene der Sprachlehrforschung existiert auch der Begriff Curriculum. Es wird von einigen Wissenschaftlern (Edmondson \& House 2005: 292) darauf hingewiesen, dass man ein Curriculum zweiartig interpretieren kann. Nach der engen Interpretation umfasst ein Curriculum bspw. eine Aufzählung von Zielen, Themen, Sprachmitteln sowie auch eine Beschreibung der Leistungen in Bezug auf die rezeptiven, produktiven, interaktiven und sprachvermittelnden Fertigkeiten, es spezifiziert aber keinen didaktischen Weg zum Erreichen dieser Ziele. So ein Curriculum ist also von einer Lehrmethode unabhängig. In Polen sind Richtlinien Curricula nach dieser engeren Interpretation, die auch als extern bezeichnet werden können, weil sie von externen Expertenkommissionen entwickelt werden. Nach denselben Autoren (ebd., 293) bezeichnet ein Curriculum im umfassenden Sinne einen didaktischen Plan, von dem folgende Angaben $\mathrm{zu}$ erwarten sind: Lehr- und Lernziele, Lehrkonzepte, Lehrprinzipien, Lehr- und Lernstrategien, Übungstypologie, Lehr- und Lernmaterialien, Medieneinsatz, sowie auch Leistungskontrolle und -bewertung.

In diesem Zusammenhang kann also festgestellt werden, dass ein internes Curriculum (Lehrplan) eine didaktische Umsetzung eines externen Curriculums (Richtlinien) zu verstehen ist. Die polnischen Richtlinien spezifizieren also die angestrebten Lernerleistungen und in den Lehrplänen werden die methodischen Vorgehensweisen erfasst, wie diese Kompetenzen erworben werden können.

\section{DAS 5-PHASEN-MODELL DER LEHRPLANARBEIT}

Die Lehrplanentwicklung vollzieht sich grundsätzlich in fünf Phasen: Reflexion, Konstruktion, Evaluation, Implementation und Revision (vgl. Pfeiffer 2001: 43; Zimmermann 1995: 135; Westphalen 1985: 45). Dieses 5-Phasen-Modell stellt eine Art der Utopie dar und ist als vollkommen und musterhaft zu betrachten, im Prinzip wird es aber mehr oder weniger ausgeprägt auf der externen und internen Ebene verwendet.

Bevor z. B. Richtlinien entstehen, findet eine Reflexionsphase statt, in der über die Sprachpolitik eines Landes und gesellschaftliche Bedürfnisse debattiert wird. Die Bedarfsanalysen, welche Sprache, von welchen Gruppen und auf welchem Niveau zu beherrschen wären, dank denen diese Fragen beantwortet werden könnten, sind eine Seltenheit (Pfeiffer 2001: 144). Abgesehen davon muss man betonen, dass von allen EU- Ländern eine gemeinsame Sprachenpolitik angestrebt wird. Über das Formulieren der Vorgaben in der Reflexionsphase entscheiden einerseits die bildungspolitischen Aspekte, andererseits Lehrbedingungen. Auf dieser Etappe werden Grobziele und Leitfäden formuliert, aufgrund derer im Weiteren die von den vom Bil- 
dungsministerium berufenen Expertenkommissionen die externen Curricula konstruieren. Die Dokumente sollten danach einer Art von Evaluation unterzogen werden, von denen die wissenschaftlich begleitete Erprobung an Versuchsschulen als die beste Methode zu empfehlen wäre (Westphalen 1985: 48). Danach wird die Phase der Implementation (d. h. der Einführung und Verbreitung) von Curricula postuliert. Die sollte sich in Verbindung mit Lehrerfortbildung, Beratung und Konkretisierung von methodischen Konzepten durch Materialentwicklung verwirklichen (vgl. Zimmermann 1995: 137; Westphalen 1985: 50). Unter der Revision kann Durchsicht und Erneuerung des Curriculums verstanden werden. Das konkrete Ziel dieser Etappe ist aber zuerst die Bewertung der Dokumente in der Praxis, die zu Korrekturen und Innovationen beitragen sollten.

Pfeiffer (2001: 143) weist darauf hin, dass man sich in Polen an diese modellhafte Vorgehensweise nicht hält. Die genannten Phasen der Lehrplanarbeiten verlaufen unkoordiniert und chaotisch. Die Revision der Richtlinien wird teilweise und indirekt durch fragmentarische Untersuchungsprojekte des Institutes der Bildungsforschung (Instytut Badań Edukacyjnych), das vom Bildungsministerium beaufsichtigt wird, durchgeführt. Die Ergebnisse der externen Prüfungen (z.B. Gymnasial- und Abiturprüfung) können auch eine Rückmeldung geben. Problematisch zu diesem Zeitpunkt scheint jedoch die nächste Schulreform zu sein, die ab 2019 die gymnasiale Stufe total abschafft. Infolgedessen entstehen neue Schultypen und die neuen Richtlinien, was zur Konsequenz hat, dass die bisherigen internen Lehrpläne sowie Lehrwerke nicht mehr aktuell sind und wieder neue entwickelt werden müssen. Die ständigen Veränderungen und die Instabilität der polnischen Bildungssituation könnten einerseits die Effizienz des Unterrichts beeinträchtigen und andererseits berufliche Verwirrung der Lehrer verursachen.

\section{CURRICULARE ARBEITEN IN DEN 70ER UND 80ER JAHREN}

Bis zu siebziger Jahren des XX. Jahrhunderts war die Fremdsprachendidaktik eher die Domäne der Praktiker, in dieser Zeit beobachtet man jedoch das Streben nach einer systematisierten, wissenschaftlichen Reflexion über Lehr- und Lernprozesse von Fremdsprachen. Die linguistischen Forschungen bildeten eine Basis für die Curriculumentwicklung, was bedeutete, dass die Grammatik und der Lernwortschatz durch die Häufigkeitszählung ermittelt wurden. Die Sprachmittel wurden auch nach dem Kriterium der Kontrastivität zur Muttersprache ausgewählt. Man kann also feststellen, dass die Grammatik, Phonologie, Morphologie und Syntax der Fremdsprachen als Progressionsprinzip in einem Lehrplan galten. 
Mitte der sechziger Jahre wurde die Fremdsprachendidaktik in Polen als wissenschaftliche Disziplin konstituiert und als Glottodidaktik bezeichnet. Wie Skowronek angibt (2013: 10), datiert man die Anfänge der Disziplin auf 1966 und verbindet sie mit Ludwik Zabrocki, der das Institut für die Angewandte Lingustik an der Adam-Mickiewicz-Universität in Poznań gegründet hat. Den Untersuchungsgegenstand der Glottodidaktik bilden Prozesse des Spracherwerbs und Sprachenlernens unter Einbezug von vielen externen und internen Faktorenkomplexen.

Mitte der siebziger Jahre waren in der polnischen Fremdsprachendidaktik die theoretischen Auseinandersetzungen zwischen dem Audiolingualismus und Kognitivismus sowie das Interesse an dem kommunikativen Ansatz sichtbar. Die Problematik der damaligen Fremdsprachendidaktik kreiste um Sprachübungen und Selbstlernen, Lehrwerke sowie auch um Leistungskontrolle. Die Überlegungen über die erwähnten Bereiche regten auch zum Nachdenken über die modernen kommunikativ ausgerichteten Lehrpläne an. Die Konstitution und die Entwicklung der polnischen Glottodidaktik, die starke Beziehungen zu Psychologie, Soziologie sowie auch zu Pädagogik zeigte, trug in späteren Zeitperioden zur Erstellung von neueren Lehrplänen (Komorowska 2017: 45) bei, wovon weiter die Rede ist.

Obwohl die Situation in Polen wegen der politischen Unruhen und des Kriegszustandes (1981) kompliziert war, wurden die curricularen Arbeiten in wissenschaftlichen Instituten fortgesetzt. Die Inspiration kam aus den linguistischen Arbeiten von Willis 1973, die auch für den Europarat bereichernd waren. Das Ziel war, die Sprachenvielfalt in ganz Europa zu fördern. Es entstanden solche Dokumente wie: „The Threshold Level for English" (1975), „Un Niveau-Senil“ (1976), „Kontaktschwelle Deutsch“ (1981). Komorowska (1999: 25) bezeichnet sie als funktionale Multiprogramme, denn: „Eine Sprache wird nach dieser funktionalen Perspektive in erster Linie als Mittel zum Ausdruck für bestimmte Zwecke charakterisiert" (Edmondon \& House 2005: 297). Sie sind universal, weil sie nicht festlegen, was und wann gelehrt oder gelernt werden sollte, sie sind ein Inventar von Zielen, Themen, Situationen, Sprachfunktionen und grammatischen Strukturen. Aufgrund dieses Rahmens entstanden in den Jahren 1984 und 1985 neue Lehrpläne für die westeuropäischen Sprachen in Grundschulen, Lyzeen und technischen Schulen ${ }^{1}$, die durch eine Verordnung des damaligen Bildungsministers eingeführt wurden. Die Lehrpläne hatten folgende Struktur: Einleitung, Bildungs- und Erziehungsziele, Bildungs- und Erziehungsinhalte, Leistungen im Bereich der vier Fertigkeiten für jede Jahrgangsstufe, morphologischlexikalischer Anhang und Anmerkungen zur Realisierung. Als Ziel nannte

${ }^{1}$ Die genannten Lehrpläne wurden in der Zeitschrift "Języki Obce w Szkole” veröffentlicht. 
man die Ausbildung der linguistischen und kommunikativen Kompetenz. Im Lehrplan findet man kaum oder nur wenige Hinweise zu Themenbereichen, Unterrichtsgestaltung oder Lehr- und Lernprozessen. Den größten Teil widmete man den Fertigkeiten, mit der Betonung auf Sprechen, Sprachmittel und Sprachfunktionen. Als Progressionsprinzipien galten weiter die Grammatik, Phonologie, Morphologie und Syntax der Fremdsprache, aber im kommunikativen Kontext.

\section{VOM ZENTRALEN LEHRPLAN ZU DEN RICHTLINIEN}

Bis zum Jahre 1989 herrschte in Polen ein zentrales Schulsystem, in dem ein nationaler Lehrplan in allen Schulen im ganzen Land obligatorisch war. Die damalige Rolle der Lehrenden beruhte also darauf, einen Stoffverteilungsplan anzufertigen und adäquate Unterrichtsmethoden zu wählen. In dem vor kurzem dargestellten Lehrplänen wurde bspw. erwähnt, dass die Lehrenden ausnahmsweise einen nicht vorgeschriebenen Stoff durchnehmen konnten. Im Verlauf der polnischen Bildungsreformdebatte nach dem Jahre 1989 begannen wichtige Diskussionen über die Lehrpläne, die früher eine präskriptive Auflistung von Zielen und Unterrichtsinhalten für jede Jahrgangsstufe enthielten (Konarzewski 2004: 13). Die Curriculumarbeiten traten in die nächste Phase ein. Gemäß Artikel 22 des Gesetzes über das Bildungssystem aus dem Jahre 1991 stellte in Polen der Bildungsminister mit einer Verordnung $u$. a. ein Mindestprogramm für fast alle obligatorischen Fächer, sowie auch Lehrpläne und Lehrwerke fest. Das erwähnte Mindestprogramm war nur eine vorübergehende Lösung und umfasste keine Regelungen für den Fremdsprachenunterricht. Die Verantwortung für die Festlegung von den Mindestinhalten wurde auf die Lernenden übertragen, die die Entscheidung treffen sollten, indem sie die bisher geltenden und vom Bildungsminister zugelassenen Lehrpläne berücksichtigen.

In dem Zeitraum von acht Jahren wurden Diskussionen über die Struktur und Ziele der zukünftigen curricularen Dokumente intensiv fortgesetzt. Die weitere Phase der Arbeiten begann 1999, als der damalige Bildungsminister Mirosław Handtke die Verordnung über die Richtlinien, die das dreistufige und reformierte Schulsystem umfassten, für die Erziehung in Kindergärten und die Allgemeinbildung in allen Schultypen, die eine Grundlage für die Entwicklung von Lehrplänen für verschiedene Fächer schaffen, unterschrieb.

In dieser Zeit war der Fremdsprachenunterricht schon ab der vierten Klasse festgeschrieben. In der Grundschule wurde nur eine Fremdsprache, im Gymnasium und Lyzeum eine zweite Fremdsprache unterrichtet. Die 
Richtlinien für den FSU von 1999 waren nur ein sehr allgemeines Dokument und hatten einen globalen Charakter, d. h. man nannte nur Aufgaben der Schule und Grobziele im Bereich der vier klassischen Fertigkeiten. Im Zeitabschnitt von 17 Jahren unterlagen die Vorgaben sehr vielen Veränderungen, wurden ständig erweitert und ausführlicher formuliert. Inzwischen (2002-2012) galt die Verordnung des Ministers für die Nationale Bildung über die Standards für externe Abschlussprüfungen in der grundschulischen, gymnasialen und postgymnasialen Stufe, die konkretisierten, über welche Kompetenzen ein Schulabgänger verfügen sollte. Seit 2012 werden die Prüfungsstandards nur von der zentralen Prüfungskomission veröffentlicht. Die curricularen Dokumente wurden mehrmals modifiziert, die bedeutenden Veränderungen an den Richtlinien für den Fremdsprachenunterricht wurden 2008 vorgenommen. Die Ziele sowie Aufgaben der Schule wurden detaillierter als bisher angegeben. Im Bereich der Fremdsprachen spiegelt sich die Fremdsprachenpolitik des Europarats wider, die zum Ziel hat, die Mehrsprachigkeit zu fördern. Als Muster werden neue Tendenzen in der Fremdsprachenvermittlung postuliert, die im Gemeinsamen europäischen Referenzrahmen für Sprachen vorhanden sind. Man führt an die Stelle der vier klassischen Fertigkeiten: Rezeption, Produktion, Interaktion und Sprachvermittlung ein und nennt obligatorische Themenbereiche für jede Bildungsetappe, die früher nicht erwähnt wurden. Die neuesten Richtlinien traten 2017 und 2018 in Kraft, als die Rückkehr zum alten dreistufigen Schulsystem in Gang gesetzt wurde. Mit der Einführung der Richtlinien für ganz Polen veränderte sich auch die Funktion der Lehrpläne, die ihre zentrale Rolle im didaktischen Prozess der Fremdsprachenvermittlung verloren, worüber im weiteren berichtet wird.

\section{LEHRPLÄNE IM DEZENTRALEN SYSTEM}

Wie schon erwähnt, war den Lehrplänen bis 1999 eine übergeordnete Funktion zugeschrieben. Ein einziger Lehrplan für ganz Polen regelte die Fremdsprachenvermittlung, d. h., dass er auch ein Steuerungsinstrument war. Der Weg zum dezentralen System bedeutete aber die Reflexion und den langsamen Übergang zu einer anderen Form der Planung, die viele pädagogische Ebenen und Faktoren wie Lehrende, Lernende, Rahmenbedingungen und Evaluationsprozesse einbezog. Seitdem die Richtlinien erstellt wurden, bekamen die Lehrer die Möglichkeit einen eigenen Lehrplan oder einen Lehrplan eines anderen Autors zu implementieren (vgl. Komorowska 2017: 87). In Bezug darauf muss jedoch erwähnt werden, dass die Lehrpläne bis 2009 weiter einer zentralen Kontrolle unterlagen und erst nach einem 
positiven Sachverständigengutachten vom Bildungsministerium erlassen wurden. Zwischen 1999 und 2009 wurden bspw. 14 DaF-Lehrpläne für die Klassen 4-6 der Grundschule erlassen, deren Autoren u.a. Waldemar Pfeiffer, Halina Stasiak, Barbara Grucza, Czeslaw Karolak waren (https:/ / men.gov.pl/ $\mathrm{pl} / z y c i e-s z k o l y /$ ksztalcenie-ogolne/podreczniki-i-programy-nauczania-dostarej-podstawy-programowej). Die von ihnen verfassten Lehrpläne resultierten aus den neuesten Curriculumsforschungen und wissenschaftlichen Theorien, die die Bedeutung der kommunikativen Kompetenz betonten. Nach dieser zehnjährigen Zeit konnten die Lehrenden für die neuen Bildungszyklen eigene Dokumente entwickeln oder die Fertigen, die von vielen Verlagen vorgeschlagen werden, realisieren. Seit dieser Zeit entscheidet der Schuldirektor mit dem Lehrerkollegium über die Zulassung der Lehrpläne, gleichzeitig trägt er die Verantwortung für die Kompatibilität der internen Lehrpläne mit den Richtlinien.

An dieser Stelle ist auch zu erwähnen, dass die Struktur eines Lehrplans bis 2014 gesetzlich geregelt war. Er sollte aus festen Komponenten bestehen: detaillierte Bildungs- und Erziehungsziele, die in den Richtlinien festgelegten Inhalte, die Art und Weise, wie diese Ziele erreicht werden, Beschreibung der erwarteten Schülerleistungen, Kriterien und Methoden zur Leistungsbeurteilung. Um in die Liste des Bildungsministeriums eingetragen werden zu können, musste so ein Lehrplan auch sachlich und didaktisch korrekt sein. Dieselben Kriterien betont Bausch (2003: 112f.) und unterscheidet sog. Kernfaktoren, die per definitionem obligatorisch sind. Erstens nennt er Leit- und Lernziele, die aus sprachenpolitischen, gesellschaftlichen und Bildungskonzepten resultieren. Sie fokussieren auf die sprachliche und interkulturelle Kompetenz, wobei sie aus vier Grundfertigkeiten besteht. Zweitens handelt es sich um Inhalte, Themen und Gegenstände. Drittens nennt er unterrichtsmethodische und handlungsorientierte Prinzipien, Arbeitstechniken, Sozialformen oder Lernstrategien. Viertens wird Lernerfolgsüberprüfung betont, in der Aspekte der Leistungsmessung und -bewertung, sowie auch des Korrekturverfahrens formuliert sind. 2014 ändert sich die gesetzliche Definition eines Lehrplans, der nach dem Bildungsgesetz eine Beschreibung darstellt, wie die Bildungs- und Erziehungsziele sowie auch Inhalte, die in den Richtlinien bestimmt sind, auf einer konkreten Bildungsetappe realisiert werden (Ustawa o systemie oświaty, art. 3).

Um die wesentlichen Angaben über die curricularen Vorgaben in Polen zu rekapitulieren, wird zum Schluss dieses Abschnitts eine chronologische Darstellung der wichtigsten Dokumente präsentiert. Die Tabelle umfasst nicht alle, sondern die bedeutendsten und im dem vorliegenden Artikel erwähnten Rechtsakte in der Zeitspanne von 1984 bis 2018, die die Praxis des Fremdsprachenunterrichts in Polen regelten. 
Tabelle 1. Übersicht der wichtigsten curricularen Vorgaben für FSU in Polen

\begin{tabular}{|c|c|c|}
\hline Datum & Vorgaben & Kommentar \\
\hline 1984 & $\begin{array}{l}\text { Lehrplan für westeuropäische Fremdsprachen (Englisch, Franzö- } \\
\text { sisch, Deutsch) für Lyzeen und Grundschulen (Erlass-Nummer } \\
08234120-29 / 84) \text {. }\end{array}$ & \multirow{6}{*}{$\begin{array}{l}\text { In diesem } \\
\text { Zeitraum } \\
\text { mussten alle } \\
\text { Lehrpläne vom } \\
\text { Minister für } \\
\text { nationale } \\
\text { Bildung erlas- } \\
\text { sen werden. } \\
\end{array}$} \\
\hline 1985 & $\begin{array}{l}\text { Lehrplan für westeuropäische Fremdsprachen (Englisch, Franzö- } \\
\text { sisch, Deutsch) für Berufslyzeen und technische Schulen (Erlass- } \\
\text { Nummer OP 23-4130-1/84). }\end{array}$ & \\
\hline 1992 & $\begin{array}{l}\text { Verordnung des Ministers für nationale Bildung Nr. } 23 \text { vom } 18 . \\
\text { August } 1992 \text { über Mindestprogramm der obligatorischen allge- } \\
\text { meinen Fächer für Grundschulen, Lyzeen und technische Schulen } \\
\text { (Amtsblatt des Bildungsministeriums 1992, Nr. 5, Pos. 25) }\end{array}$ & \\
\hline 1999 & $\begin{array}{l}\text { Verordnung des Ministers für nationale Bildung vom 15. Februar } \\
1999 \text { über Richtlinien der Allgemeinbildung (Gesetzblatt 1999, } \\
\text { Nr. 14, Pos. 129). }\end{array}$ & \\
\hline 2001 & $\begin{array}{l}\text { Verordnung des Ministers für nationale Bildung vom 10. August } \\
2001 \text { über Standards für externe Abschlussprüfungen in allen } \\
\text { Schultypen (Gesetzblatt 2001, Nr. 92, Pos. 1020), galt mit späteren } \\
\text { Änderungen bis 2012. }\end{array}$ & \\
\hline 2008 & $\begin{array}{l}\text { Verordnung des Ministers für nationale Bildung vom 23. Dezem- } \\
\text { ber } 2008 \text { über Richtlinien der Kindergartenerziehung und der } \\
\text { Allgemeinbildung in den jeweiligen Schultypen (Gesetzblatt 2009, } \\
\text { Nr. 4, Pos. 17). }\end{array}$ & \\
\hline $\begin{array}{l}\text { seit } \\
2012\end{array}$ & \multicolumn{2}{|c|}{$\begin{array}{l}\text { Informationen über die Prüfungsstandards werden nur von der zentralen Prü- } \\
\text { fungskommission veröffentlicht. }\end{array}$} \\
\hline 2017 & \multicolumn{2}{|c|}{$\begin{array}{l}\text { Verordnung des Ministers für nationale Bildung vom 14. Februar } 2017 \text { über Richtli- } \\
\text { nien der Kindergartenerziehung und der Allgemeinbildung in Grundschulen, Be- } \\
\text { rufsschulen ersten Grades und Postlyzealen Schulen (Gesetzblatt 2017, Pos. 356). }\end{array}$} \\
\hline 2018 & \multicolumn{2}{|c|}{$\begin{array}{l}\text { Verordnung des Ministers für nationale Bildung vom 30. Januar } 2018 \text { über Richtli- } \\
\text { nien der Allgemeinbildung in Lyzeen, technischen Schulen und Berufsschulen } \\
\text { zweiten Grades (Gesetzblatt 2018, Pos. 467). }\end{array}$} \\
\hline
\end{tabular}

\section{RICHTLINIEN UND LEHRPLÄNE FÜR DAF-UNTERRICHT IM LICHTE EINER PILOTUNTERSUCHUNG}

Ziel der Untersuchung war die Erhebung der Informationen von Sprachlehrern im DaF-Unterricht zur Funktion der Richtlinien und Lehrpläne in der schulischen Praxis. Sie war auf die Strategie der Entwicklung von Lehrplänen und die Wahl der Lehrwerke gezielt. Im Rahmen einer Onlinebefragung im Mai 2018 wurden ungefähr 100 DaF-Lehrer/innen aller Bildungsstufen eingeladen, an der Befragung teilzunehmen. Es wurden 26 Fragebögen ausgewertet, was einer Rücklaufquote von $26 \%$ entspricht. In dem ersten Teil des Fragebogens wurden die Fragen zur der Bildungsetappe, auf der die 
Lehrer/innen tätig sind, zur Lehrerbeförderungsstufe und zum Ort der Arbeit gestellt. Fünf von ihnen arbeiten ausschließlich an einer postgymnasialen Schule und zwei andere nur an einem Gymnasium. 19 der Befragten unterrichten an verschiedenen Schultypen. 26 Personen üben ihren Beruf in Pommern z. B. in Słupsk, Gdansk, Wejherowo, Malbork, Kościerzyna und anderen kleineren Orten aus, eine von ihnen in Westpommern. Der überwiegende Teil sind Diplom- und ernannte Lehrer/innen (fast 77\%), die anderen dagegen sind auf Referendarzeit (11,5\%) oder Vertragslehrer/innen $(11,5 \%)$.

Im weiteren Teil wurde danach gefragt, ob die Lehrer/innen eigene Lehrpläne oder Lehrpläne der anderen Autoren, die von den Sprachverlagen vorgeschlagen werden, in die Praxis einsetzen und welche Gründe dafür stehen, solche Entscheidungen zu treffen. Die Probanden wurden auch gebeten die Titel, Verlage und Autoren zu nennen. Aus der Bewertung geht hervor, dass 12 der Befragten die fertigen Lehrpläne ohne jegliche Veränderungen bevorzugen, die weiteren 10 auch die von den anderen verfassten Lehrpläne realisieren, indem sie sie an einigen Stellen modifizieren. Die Veränderungen halten sie für notwendig, um die Dokumente sowohl an die Bedürfnisse der Schüler, an die vorhanden Stundenzahl des Deutschunterrichts an ihren Schulen anzupassen, als auch um einige sachliche Fehler zu korrigieren. Nur 9 von 22 der Befragten, die von anderem Autor verfasste Curricula realisieren, gaben die Titel an. Die Lehrer/innen stützen sich in ihrer didaktischen Praxis auf die Lehrpläne von folgenden Verlagen: Nowa Era, Wydawnictwa Szkolne i Pedagogiczne, PWN Wydawnictwo Szkolne, LektorKlett, Pearson. Auf die Frage, warum sie die fertigen Lehrpläne bevorzugen, gaben $74 \% \mathrm{zu}$, eine Sicherheit der Kompatibilität der Lehrpläne sowohl mit den Richtlinien als auch mit den Lehrwerken zu haben. 36\% dagegen haben keine Erfahrung, solche Dokumente zu erstellen. Lediglich 4 der Befragten gaben an, eigene interne Curricula $\mathrm{zu}$ implementieren, was damit zusammenhängt, dass sie Innovationen verwirklichen. Fast alle stellten fest, den Inhalt der Lehrpläne sehr gut oder gut zu kennen, obwohl, wie schon früher erwähnt, nur ein Drittel den Titel oder den Autor wusste.

Der nächste Fragenkomplex befasste sich mit dem Zeitpunkt, zu welchem Lehrpläne und Lehrwerke für den nächsten Bildungszyklus gewählt werden. Es zeigte sich, dass 20 der Befragten ihre Wahl hinsichtlich der Lehrpläne vor dem Anfang eines Schuljahres, ohne ihre Gruppen kennenzulernen, treffen. Demgegenüber stehen nur 6, die darüber erst im September entscheiden. Was das Problem der Lehrwerke anbelangt, werden sie von den 17 Befragten vor der Entscheidung über die Lehrpläne gewählt.

Darauffolgend wurde ermittelt, zu welchen Zwecken die Curricula benutzt werden und welche Funktion sie in der didaktischen Planung erfüllen. 
$42 \%$ der Befragten finden Lehrpläne als unentbehrliche Dokumente, die die didaktische Arbeit systematisieren. 38,5\% betrachten sie als einen methodischen Wegweiser, 23\% dagegen brauchen keinen Lehrplan, wenn sie ein vom Bildungsministerium zugelassenes Lehrwerk haben, das eine Garantie der Kompatibilität mit den Richtlinien gibt. In Bezug darauf stellten 15\% fest, dass ein Lehrplan nichts Besonderes in ihr didaktisches Handeln mit sich bringt. Es zeigte sich jedoch, dass ein Curriculum eine bedeutende Rolle bei den folgenden Lehrertätigkeiten spielt: Stoffverteilungsplan erstellen $(80 \%)$, Unterrichtseinheiten planen $(69 \%)$ oder Bewertungskriterien formulieren (58\%). 21 der Befragten gaben auch an, die Evaluation der Lehrpläne nach einem geschlossenen Bildungszyklus durchzuführen.

Zum Schluss wurden die Lehrenden gebeten, eine Hierarchie der Dokumente und Materialien zu erstellen. An der ersten Stelle stehen Richtlinien, nachfolgend wurden Lehrwerke genannt, weiterhin platzieren die Befragten die Prüfungsstandards, die in den veröffentlichten Bestimmungen der zentralen Prüfungskommission aufgelistet sind. An den zwei letzten Stellen wurden Stoffverteilungs- und Lehrpläne erwähnt.

\section{SCHLUSSFOLGERUNGEN}

Wie die Ergebnisse der Pilotuntersuchung zeigen, betonen die Lehrer/ innen zwar die Wichtigkeit der internen Curricula in der Planung des didaktischen Prozesses, aber als erstes wird ein Lehrwerk gewählt. Unterzieht man einige in der Untersuchung genannten Lehrpläne einer kurzen Analyse, sieht man gleich, dass einige von ihnen lehrwerkabhängig sind, was bedeutet, dass sie erst nach dem Verfassen der Lehrwerke erstellt wurden. Zu denen kann man die Lehrpläne von Kubicka oder von Wawrzyniak für das Gymnasium (LektorKlett), von Niebrzydowska für die Lyzeen und technischen Schulen (LektorKlett), von Zenderowska-Korpus (LektorKlett) für die postgymnasialen Schulen zählen. In all diesen Curricula findet man Vorschläge für Lehrwerke und beispielhafte Stundenentwürfe, die mit dem Lehrwerk des Verlags integriert sind. Trotzdem ist festzuhalten, dass sie die Progression der Inhalte nicht festlegen, sondern ein Inventar von Themenbereichen, Sprachmitteln, Sprachfunktionen sowie grammatischen Strukturen als Leistungen darstellen, die nach dem Bildungszyklus zu erwarten sind. Dies gibt auch die Möglichkeit, sie nach kleinen Modifikationen mit ganz anderen Lehrmaterialien zu implementieren. In diesem Fall hat man jedoch mit keiner optimalen, sondern mit einer restriktiven Strategie der Planung zu tun. Für optimal kann man eine Situation halten, in der der oder die Lehrende zuerst seine oder ihre Gruppe kennenlernt und erst dann den Bedürf- 
nissen und intellektuellen Möglichkeiten angemessen einen Lehrplan erstellt, auswählt oder modifiziert, der alle Kernkomponenten: Ziele, Inhalte, Methoden und Lehrmittel umfasst. Hier muss aber erwähnt werden, dass Edmondson und House (2006: 292) eine Curriculumentscheidung einer Lehrwerkentscheidung gleichsetzen: „(...) und zwar in dem Sinne, daß (in einem Lehrwerk) eine strukturierte Sequenz von Informationen und Aufgaben vorgelegt wird, die Lehrer im Unterricht systematisch einsetzen können“.

Im Vordergrund aller curricularen Entscheidungen auf der internen Ebene stehen jedoch die Richtlinien, die den ganzen Unterrichtsprozess vermutlich stark beeinflussen. Konarzewski (2004: 16f.) betont, dass dieses staatliche Dokument ein Hauptinstrument ist, das den Regierenden die Bildungspolitik im dezentralen System zu führen, ermöglicht. Es beinhaltet globale Ziele und grundsätzliche Inhalte für verschiedene Fächer, u.a. für den Fremdsprachenunterricht, und in dieser Funktion richtet es sich an Lehrplan- und Lehrwerkautoren sowie auch an die Verfasser der externen Prüfungen. Alle Lehrer/innen sind auch Rezipienten, die für die Kompatibilität der realisierten Lehrpläne mit den Rahmenrichtlinien zuständig sind. Die Richtlinien erheben also einen Anspruch auf Rechtsverbindlichkeit und Allgemeingültigkeit und gelten als globale Steuerungsinstrumente des Schulsystems, führen gleichzeitig zu einer optimalen Vergleichbarkeit der Leistungen im ganzen Land. Neben der spezifischen Steuerungsfunktion nennt Neuner (2001: 799) auch ihre allgemeine Orientierungsfunktion und hebt hervor, dass sie dadurch den Lehrenden mehr Freiräume bei der Wahl oder Erstellung von Lehrplänen geben, sowie auch den Entscheidungs- und Gestaltungsmöglichkeiten im Unterrichtsprozess dienen sollten (vgl. Westphalen 1985: 13).

An dieser Stelle kann man feststellen, dass die polnischen Rahmenrichtlinien als bildungspolitisches Programm gelten, die der Planung, Steuerung, Optimierung und der Erfolgskontrolle dient. Die Lehrpläne spielen dabei nach wie vor eine wichtige Rolle, sie legen keine Progression mehr fest, sondern beschreiben, wie man die sprachlichen Kompetenzen und Fertigkeiten entwickeln kann. Einerseits sind sie offen und flexibel, können erweitert, verkürzt oder modifiziert werden, je nach den Bedürfnissen und Lehrbedingungen, andererseits aber enthalten alle von ihnen einen gemeinsamen Orientierungsrahmen, der den Fremdsprachenunterricht in unserem Land optimiert.

Ein Ziel dieses Beitrags war es, die Evolution vom zentralen bis zum dezentralen Planen des Fremdsprachenunterrichts in Polen zu schildern. Die Analysen der Rechtsakte sowie die Ergebnisse der Pilotuntersuchung rechtfertigen die Aussage, dass die heutigen Umstände den polnischen Fremdsprachenlehrer/innen viel Autonomie für die Entwicklung der internen 
Curricula gewährleisten, obwohl die meisten Befragten (fast 85\%) die Lehrpläne der anderen Autoren in die Praxis einsetzen. Im Anschluss daran lassen sich drei Postulate für die Aus- und Weiterbildung der Lehrer ableiten. Erstens sollte das jetzige Modell der Fremdsprachenlehrerausbildung in Bezug auf die Aspekte der Curriculumentwicklung stärker reflektiert werden. Zweitens wäre es wirklich empfehlenswert Lehramtsstudierenden praktisches Können beizubringen, wie Lehrpläne für FSU erstellt und evaluiert werden könnten. Drittens sollten sowohl Fortbildungsorganisatoren als auch Lehrbuchverlage Kurse und Schulungen in diesem Bereich der Fremdsprachendidaktik in ihr Angebot einbeziehen.

\section{LITERATURVERZEICHNIS}

Bausch, K.-R. (2003). Funktionen des Curriculums für das Lehren und Lernen fremder Sprachen. In: E. Burwitz-Melzer / C. Riemer / K.-R. Bausch / H.-J. Krumm (Hrsg.), Handbuch Fremdsprachenunterricht, 4. Auflage (S. 111-115). Tübingen: A. Francke.

Christ, I. (2016). Staatliche Regelungen für den Fremdsprachenunterricht: Curricula, Richtlinien, Lehrpläne. In: E. Burwitz-Melzer / G. Melhorn / C. Riemer / K.-R. Bausch / H.-J. Krumm (Hrsg.), Handbuch Fremdsprachenunterricht, 6. Auflage (S. 56-60). Tübingen: A. Francke.

Edmondson, W. / House, J. (2005). Einführung in die Sprachlehrforschung. Tübingen und Basel: A. Francke.

Funk, H. (2016). Curriculare Entwicklungsprinzipien. In: E. Burwitz-Melzer u. a. (Hrsg.), Handbuch Fremdsprachenunterricht, 6. Auflage (S. 151-156). Tübingen: A. Francke.

Komorowska, H. (1999). O programach prawie wszystko. Warszawa: WSiP.

Komorowska, H. (2017). Ksztatcenie jezykowe w Polsce. 60 lat czasopisma "Jezyki Obce w Szkole". Warszawa: Fundacja Rozwoju Systemu Edukacji, http://jows.pl/sites/default/files/ ksztalcenie_jezykowe_w_polsce_www.pdf

Konarzewski, K. (2004). Reforma oświaty. Podstawa programowa i warunki kształcenia. Warszawa: Instytut Spraw Publicznych.

Kubicka, A. (2009). Program nauczania jezyka niemieckiego w klasach I-III gimnazjum. Poziom III.O dla początkujących. Poznań: LektorKlett.

Neuner, G. (2001). Curriculumentwicklung und Lehrziele Deutsch als Fremdsprache. In: G. Helbig / L. Götze / G. Henrici / H.-J. Krumm (Hrsg.), Deutsch als Fremdsprache. Ein internationales Handbuch (S. 797-809). Berlin: Walter de Greuter.

Niebrzydowska, B. (2017). Program nauczania języka niemieckiego dla klas I-III liceum ogólnoksztatcacego i klas I-IV technikum IV etap edukacyjny (poziom IV.0 - dla początkujacych i poziom IV.1.P - dla kontynuujących naukę). Poznań: LektorKlett.

Pfeiffer, W. (2001). Nauka języków obcych. Od praktyki do praktyki. Poznań: Wagros.

Rozporządzenie Ministra Edukacji Narodowej i Sportu z dnia 5 lutego 2004 r. w sprawie dopuszczania do użytku szkolnego programów wychowania przedszkolnego, programów nauczania i podręczników oraz cofania dopuszczenia (Dz.U. 2004, nr 25, poz. 220).

Rozporządzenie Ministra Edukacji Narodowej i Sportu z dnia 26 lutego 2002 r. w sprawie podstawy programowej wychowania przedszkolnego oraz kształcenia ogólnego w poszczególnych typach szkół (Dz.U. 2002, nr 51, poz. 458). 
Rozporządzenie Ministra Edukacji Narodowej z dnia 30 stycznia 2018 r. w sprawie podstawy programowej kształcenia ogólnego dla liceum ogólnokształcącego, technikum oraz branżowej szkoły II stopnia (Dz.U. 2018, poz. 467).

Rozporządzenie Ministra Edukacji Narodowej z dnia 14 lutego 2017 r. w sprawie podstawy programowej wychowania przedszkolnego oraz podstawy programowej kształcenia ogólnego dla szkoły podstawowej, w tym dla uczniów z niepełnosprawnością intelektualną w stopniu umiarkowanym lub znacznym, kształcenia ogólnego dla branżowej szkoły I stopnia, kształcenia ogólnego dla szkoły specjalnej przysposabiającej do pracy oraz kształcenia ogólnego dla szkoły policealnej (Dz.U. 2017, poz. 356).

Rozporządzenie Ministra Edukacji Narodowej z dnia 15 lutego 1999 r. w sprawie podstawy programowej kształcenia ogólnego (Dz.U. 1999, nr 14, poz. 129).

Rozporządzenie Ministra Edukacji Narodowej z dnia 15 lutego 1999 r. w sprawie warunków i trybu dopuszczania do użytku szkolnego programów nauczania z zakresu kształcenia ogólnego oraz warunków i trybu dopuszczania do użytku szkolnego podręczników i zalecania środków dydaktycznych (Dz.U. 1999 nr 14, poz. 130).

Rozporządzenie Ministra Edukacji Narodowej z dnia 21 maja 2001 r. w sprawie podstawy programowej wychowania przedszkolnego, kształcenia ogólnego $\mathrm{w}$ poszczególnych typach szkół oraz kształcenia w profilach w liceach profilowanych (Dz.U. 2001, nr 61, poz. 625).

Rozporządzenie Ministra Edukacji Narodowej z dnia 8 czerwca 2009 r. w sprawie dopuszczania do użytku w szkole programów wychowania przedszkolnego i programów nauczania oraz dopuszczania do użytku szkolnego podręczników (Dz.U. 2009, nr 89, poz. 730).

Rozporządzenie Ministra Edukacji Narodowej z dnia 23 grudnia 2008 r. w sprawie podstawy programowej wychowania przedszkolnego oraz kształcenia ogólnego w poszczególnych typach szkół (Dz.U. 2009, nr 4, poz. 17).

Skowronek, B. (2013). Glottodidaktik und Fremdsprachenunterricht in der Diskussion. Poznań: Wydawnictwo Naukowe UAM.

Ustawa z dnia 7 września 1991 r. o systemie oświaty (Dz.U. 1991, poz. 425 z późn. zm.).

Ustawa z dnia 14 grudnia 2016 r. Prawo Oświatowe (Dz.U. 2017, poz. 59 z poźn. zm.).

Wawrzyniak, M. (2015). Program nauczania jezzyka niemieckiego dla klas I-III gimnazjum. Poziom III.0. Poznań: LektorKlett.

Westphalen, K. (1985). Lehrplan - Richtlinien - Curriculum. Stuttgart: Klett.

Zarządzenie nr 23 Ministra Edukacji Narodowej z dnia 18 sierpnia 1992 r. w sprawie minimum programowego obowiązkowych przedmiotów ogólnokształcących (Dz.U. 1992, nr 5, poz. 25).

Zenderowska-Korpus, G. (2012). Program nauczania języka niemieckiego dla klas I-III liceum ogólnoksztatcacego i klas I-IV technikum. Poznań: LektorKlett.

Zimmermann, G. (1995). Das sprachliche Curriculum. In: K.-R. Bausch u.a. (Hrsg.), Handbuch Fremdsprachenunterricht, 3. Auflage (S. 135-142). Tübingen: Francke.

Received: 13.06.2018; revised: 29.08.2018 
